\title{
An Improved Segmentation Method for Spray Painting Characters on Slab Surface
}

\author{
Chun-Hui HUANG ${ }^{1}$, Qi-Jie ZHAO ${ }^{1,2, a^{*}}$, Zhen-Nan KE${ }^{1}$, Xian-Fa LI ${ }^{1}$ \\ ${ }^{1}$ School of Mechatronic Engineering and Automation, Shanghai University, 200072, China \\ ${ }^{2}$ Shanghai Key Laboratory of Intelligent Manufacturing and Robotics, 200072, China \\ azqj@shu.edu.cn \\ Corresponding author
}

\begin{abstract}
Keywords: Slab Surface; Painting Character; Image Processing; String Location; Character Segmentation.
\end{abstract}

\begin{abstract}
In order to improve segmentation of spray painting character in the slab surface, in this paper, we propose to improve the picture quality by image enhancement and filtering, segmenting image from coarse to fine to solve problem in character image segmentation stage step by step. Local dynamic thresholding method is constructed for string localization. Character segmentation algorithm based on morphology, connected component analysis and secondary segmentation to solve the problem of adhesion and fracture present in the images collected in industrial locale environment. Filter with mask used for protecting characters is proposed to solve thin line noise problems. We establish an experimental system to study, results shows that the method has a good effect on adhesion, fracture type and line-interfered image.
\end{abstract}

\section{Introduction}

Painting mark of billet, realizing the automation of production and logistics by tracking and recognition of identification characters has attracted extensive attention of steel enterprise [1]. Due to the high-temperature danger and other situation's risking which is not conducive to manual work, recognition of characters on the billet's surface with image capturing by camera has a wonderful prospect $[2,3]$. Spray painting characters, which has frequent occurrence of adhesion and fracture, is different from printed characters on scanned documentation. So segmentation of identification characters from images correctly is of great benefit to improving character recognition rate.

To overcome this issue, a lot of relevant research is being done. A. R. Alexandria construct a system to recognize characters on energy meters [4]. SungHoo Choi et al. present a character location and segmentation method, which is based on edge detection and image feature extraction [5]. Hanyu HONG et al. have proposed a kind of character location method based on projective invariant features to recognize characters of billet under complex lighting effectively [6]. In the preliminary work, we have proposed a segmentation method to recognize mark of billet, which took advantage of color information to locate and then tilt correct the characters of the image [3]. These studies are of valuable reference in the billet's spray printing characters segmentation. However, splitting and touching of the spray painting characters, the line noise jamming etc. are still the major problems of character segmentation and lead to mis-recognition due to the complicated conditions of steel productions and mark printings [7-9].

Based on the preliminary works, this study focuses on the segmentation of spray painting characters on slab surface. And according to the features of images' characters, we provide an image enhancement algorithm and a filter algorithm, which are aim to improve the effect of segmentation of spray painting characters with adhesion, fracture or line noise.

\section{Proposed Methodology}

In order to implement character segmentation for spray painting characters on slab surface, an orderly single character set are required for character recognition. Proposed image segmentation 
method or detail procedure is illustrated in Fig. 1, which mainly includes of image preprocessing, string localization and character segmentation. In image preprocessing stage, image enhancement is implemented. In string location stage, coarse string localization using light-insensitive local dynamic adaptive thresholding is implemented, which provides a basis for single-character segmentation. In single-character segmentation stage, the Morphology using common structure and specific structure are respectively used, and CCA, Secondary Segmentation, image de-noising are used to realize fine string localization and accepted orderly single character set.
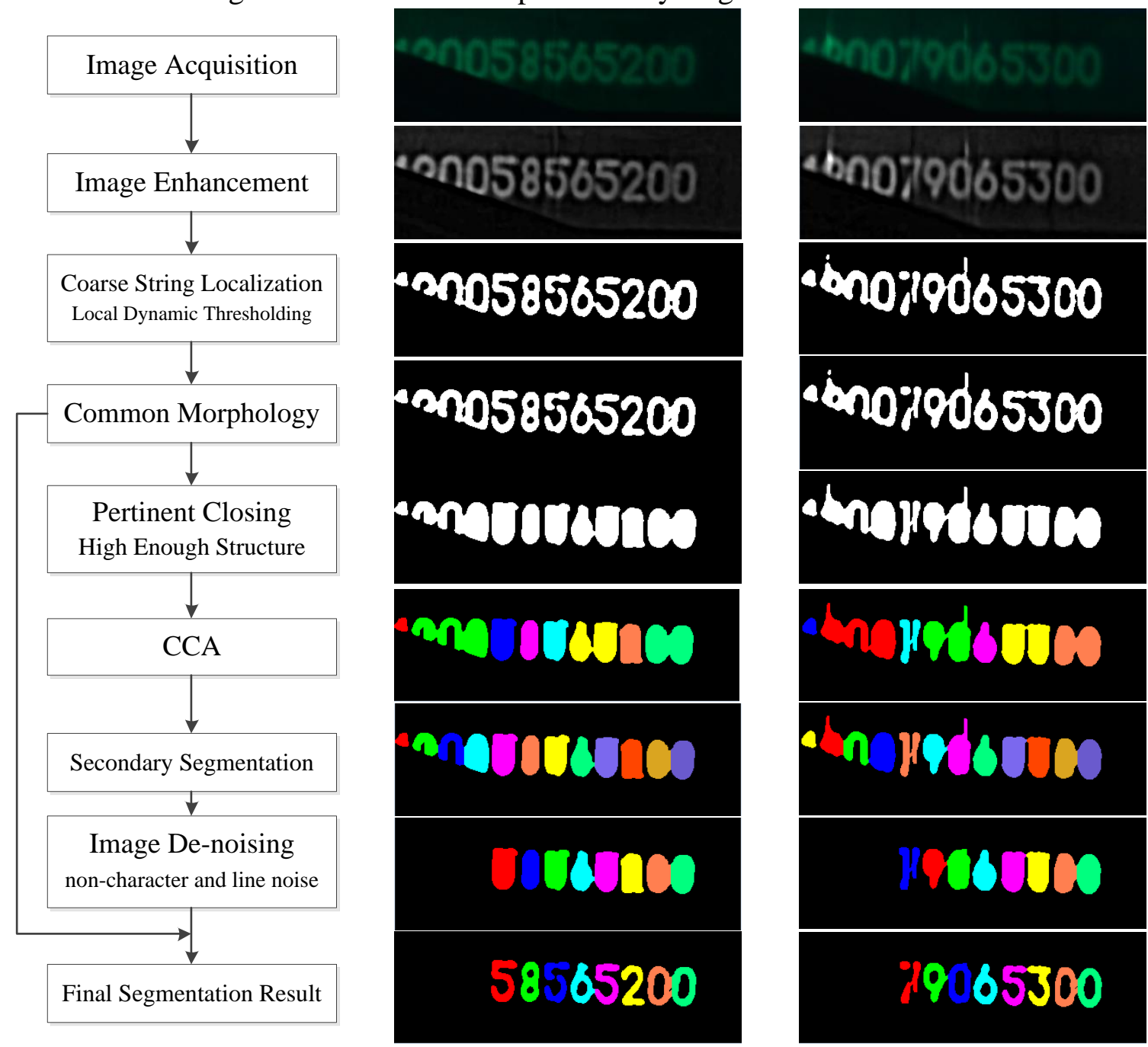

Fig. 1 character segmentation method

\section{Detail of Proposed Method}

\section{Image Pre-Processing and String Locating}

In Image pre-processing stage, the detail task is to enhance image. First, images are transformed to gray scale image to remove redundant information, then local dynamic adaptive enhancement algorithm based on gray value transformation is constructed to solve segmentation problem caused by poor image contrast, and finally median filter smooth character information, filtered random noise interference.

The local dynamic adaptive enhancement algorithm is show in Eq. 1. The algorithm implements via expending difference between gray value of a pixel and local region with the pixel as center with size of $w_{m} \times h_{m}$. In detail, first, the algorithm carries out mean filter get mean gray value of local region $g_{\text {mean }}$, and then calculate the result $g_{\text {res }}$ using factor which is intensity of contrast emphasis serving as measurement of the increase in contrast. Besides, $w_{m}$ and $h_{m}$ is determined by the width of character. 


$$
g_{\text {res }}=\operatorname{round}\left(\left(g_{r, c}-g_{\text {mean }}\right) \times \text { factor }\right)+g_{r, c}
$$

The local dynamic adaptive thresholding algorithm as shown in Eq. 2 is designed to locating String preliminarily. The difference between foreground and background is extracted to determine foreground region or character region. The background is extracted by a mean filter, the size of the filter determines the size of objects which can be segmented, so size of kernel of mean filter are valued according to the size of character. A mean filter is constructed to obtain $g_{\text {back }}$ which is the grayvalue of local background. The difference between $g_{r, c}$ and $g_{\text {back }}$ is taken as feature of segmentation. All pixels belonging to Image $I$ with difference bigger than threshold $g_{\text {diff }}$ constitute character region $R$, which represents position of String.

$$
R=\bigcup\left\{p(r, c) \in I|| g_{r, c}-g_{\text {back }} \mid \geq g_{\text {diff }}\right\}
$$

\section{Character Segmentation and Repairing}

A processing algorithm based on Morphology is designed for Region $R$ extracted by above thresholding. First, opening operation in Region Morphology with rectangle structure element is used to smooth rough skeleton in $R$. Then, closing operation with rectangle structure element and slant rectangle structuring element is used to repair slight fracture and get $R \_s t r i n g$. Then closing operation with rectangle whose height equals to height of character and whose width equals to 0 is used for $R_{-}$string to repair severe fracture. Then conduct CCA to extract connected regions, segment single character region set preliminarily, getting single character region set $R \_$chars 1 []. Then Secondary Segmentation is used to separate touched characters. Finally, we get intersection with $R \_$string being opened with a rectangular structuring element to get $R \_$chars2[]. Effect of algorithm to handle adhesion and fracture is shown in Fig. 2.

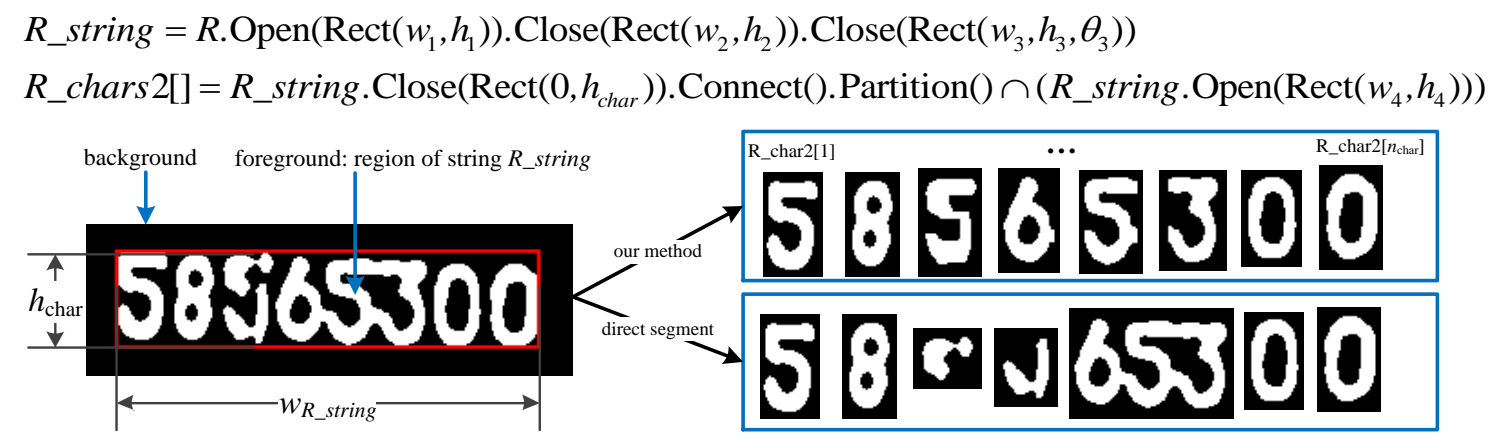

Fig. 2 effect of handling adhesion and fracture

The above-mentioned Secondary Segmentation algorithm is implemented as follow. Mean width of characters $w_{\mathrm{m}}$ is calculated by width of string $w_{\mathrm{R}_{-} \text {string }}$ and number of character $n_{\text {char }}$ with equation $w_{\mathrm{m}}=w_{\mathrm{R}_{-} \text {string }} / n_{\text {char }}$. Width of each region in region $\operatorname{set}(w[])$ is counted by feature extracting. Then the algorithm calculates quotient of $w[i]$ and $w_{\mathrm{m}}$, and round the result to the nearest integer to get $\mathrm{n}, \mathrm{n}=\operatorname{round}\left(w[\mathrm{i}] / w_{\mathrm{m}}\right)$, which is used to judge whether there are adhesion issues through compare $\mathrm{n}$ to 1 . As shown in Eq. 4, vertical projection is used to get value of $f(t)$ and find valley to determine the position of Secondary Segmentation.

$$
\begin{aligned}
& \forall(r, c) \in I, \chi(r, c)=\left\{\begin{array}{l}
1,(r, c) \in R_{-} \text {charl } \\
0,(r, c) \notin R \_ \text {char } 1
\end{array}\right. \\
& f(\mathrm{t})=\sum_{r=1}^{h} \chi\left(r, c_{t}\right)(\mathrm{t}=\{-\mathrm{k}, \ldots, 0,1, \ldots, \mathrm{k}\}) \\
& l=\underset{t}{\operatorname{argmin}} f(t)
\end{aligned}
$$


A filter algorithm as shown in Eq. 5 through mask to protect character is proposed to solve problem caused by line noise. First, the algorithm combine $R_{-}$char[] into a region. Then, close operator with structuring element with enough width is used to integrate a closed object, to ensure that there are significant differences between character and line noise. Then, open operator with structuring element with enough width is used to filter line. Finally, $R \_c h a r 2[]$ is intersected with region without line noise to recover character structure.

$$
R \_ \text {chars3[] }=R \_ \text {chars2[] } \cap\left(R \_ \text {chars2[].Union }() . \operatorname{Close}\left(\operatorname{Rect}\left(w_{5}, 0\right)\right) . \operatorname{Open}\left(\operatorname{Rect}\left(w_{6}, 0\right)\right)\right)
$$

\section{Experiment}

To demonstrate the effectiveness and the commonality of this design method mentioned in the article, some experiments have been done in the steelmaking plant. Experiment environment and equipment and the collected image samples are shown in Fig.3. The equipment mainly includes a metal spray marking machine with multi nozzles, one color camera SQ-S20C-H30, dustproof, waterproof and Anti-high Temperature Metal Halide Lamp that the spectrum of the lighting is close to sunlight, a set of MIDOPT FS100 band-pass filters, a computer (with CPU 3.0, Memory 2 GB). Our acquisition image resolution is $400 \times 130$. The position size of 8 recognized digital characters is $235 \times 50$. As is shown in Fig. 3 , the acquired images has occurrence of low-contrast, adhesion, fracture and line interference etc.
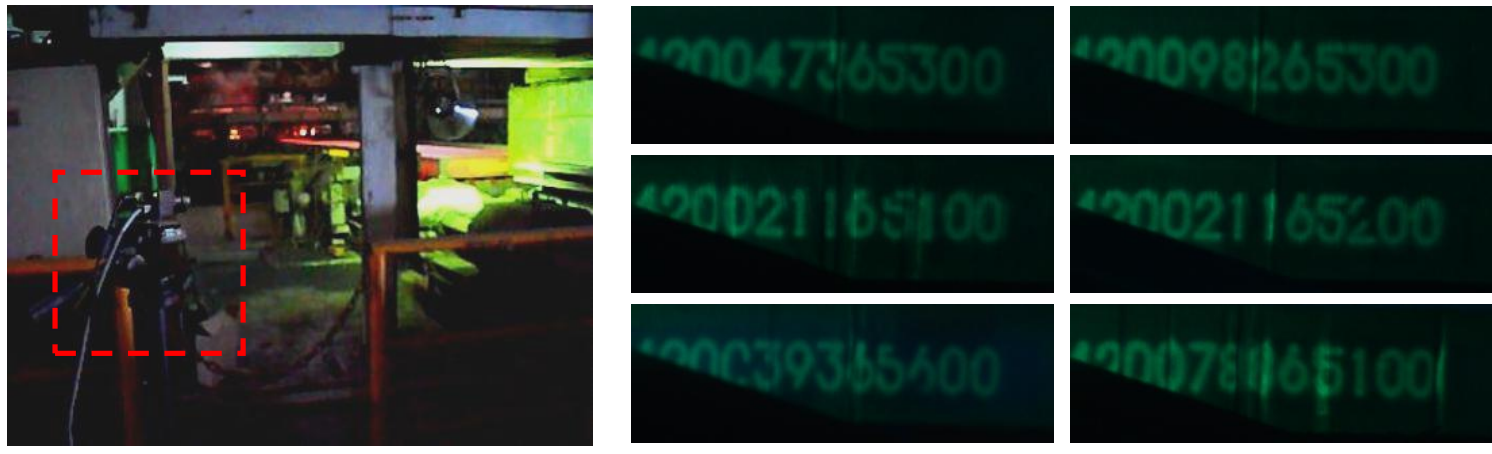

Fig. 3 experimental environment and instances of experimental samples
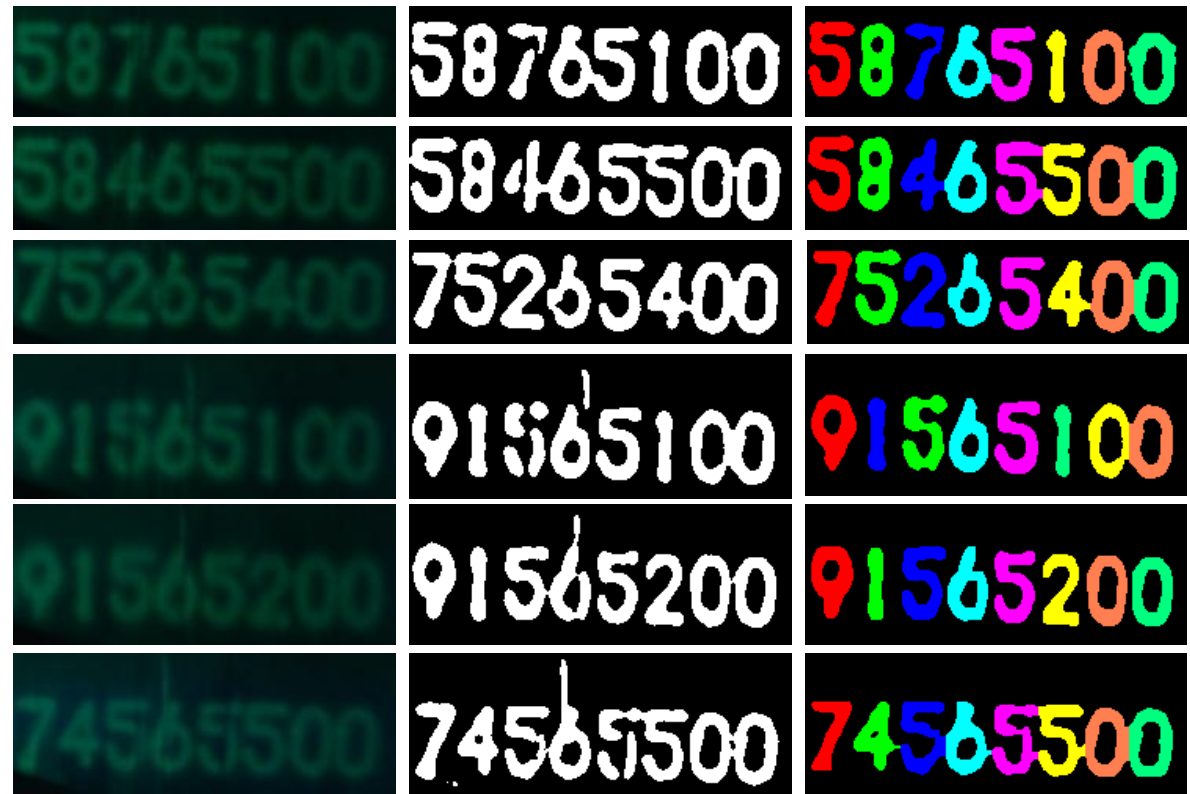

Fig. 4 segmentation results of image with adhesion, fracture or line noise 
According to the method mentioned above, we designed a project of image segmentation software to deal with 1425 samples with adhesion, fracture or line noise. The segmentation software interface contains an image displaying region, the software stores the result in database. Fig.4 shows the processed results of problems. Among the figures, the left ones are original images, the middle ones are corresponding results of string localization, and the right ones are the results of image segmentation that marks the characters in different colors.

As fig. 4 shows, the characters " 7 " and " 4 " in the first two images are split obviously due to the printing quality problems, and are finally correctly segmented by our method. Besides, the characters which are connected or influenced by line noise obviously are finally correctly segmented by our method. All of the samples all are with defects, and we get 1408 samples (98.8\%) segmented correctly. The experiment result suggests the proposed character segmentation method could effectively and efficiently segment the digital characters marked on the billets.

\section{Conclusion}

Improving manufacturing information management and control has important significance for productivity increase and cost reduction, which is also very meaningful for improving the company's competitiveness. This study focuses on the information extraction of material coding in information system operations of steel enterprises. The encoded massage of character on slab surface is remotely acquired using machine vision. First, combined with spray painting characters' characteristics, we provide an adaptive enhancement algorithm and filter to pre-process images effectively. A method insensitive to illumination conditions is proposed to locate characters, which is based on difference between foreground and background. In order to solve the problems including adhesion, fracture or line noise, our study carries out a processing method based on CCA, enhanced morphology algorithm, secondary segmentation using projection, and line noise processing algorithm based on mask protecting. These methods segment the spray printing characters from coarseness to fine and paly a very great part of this process. The result shows the effective segmentation of spray printing characters on billet and demonstrates the applicability of this design method mentioned above.

\section{References}

[1] C G Deng, Z Q Xiang, J C Xie, et al, Design and study on marking system online for steel products, Computer Integrated Manufacturing Systems, vol. 16, no. 9, (2010), 1859-1865.

[2] Q J Zhao, P Cao and D W Tu. Toward intelligent manufacturing: label characters marking and recognition method for steel products with machine vision. Advances in Manufacturing, vol. 2, no. $1,(2014), 3-12$.

[3] Q J Zhao, P Cao and Q X Meng, Image Capturing and Segmentation Method for Characters Marked on Hot Billets, Advanced Materials Research, vol. 945-949, (2014), 1830-1836.

[4] A R Alexandria, P C Cortez, J H S Felix, et al. An OCR System for Numerals Applied to Energy Meters. IEEE Latin America Transactions, vol. 16, no. 9, (2014), 957-964.

[5] S H Choi, J P Yun, K Koo and W K Sang. Localizing slab identification numbers in factory scene images, Expert Systems with Applications, vol. 36, no. 9, (2012), 7621-7636.

[6] H Y Hong, B Dong, Y Shi, et al. Research on Billet Character Positioning Based on the Projective Invariants, Software Guide, vol. 2, (2015), 145-148.

[7] A Rebelo, J S Cardoso, Staff Line Detection and Removal in the Grayscale Domain, 2013 12th International Conference on Document Analysis and Recognition (Icdar), Washington, DC, (2013).

[8] A Namane, A Guessoum, EH Soubari, P Meyrueis. CSM neural network for degraded printed character optical recognition, Journal of Visual Communication and Image Representation, vol. 35, 
no. 5, (2014), 1171-1186.

[9] L Mennillo, J Cousty, L Najman. A Comparison of Some Morphological Filters for Improving OCR Performance", Mathematical Morphology and Its Applications to Signal and Image Processing. Springer International Publishing, vol. 9082, (2015), 134-145.

[10]S Heamawatanachai, K Chaemthet, T Changpan. Development of a Machine-Vision System for Recording of Force Calibration Data, International Journal of Modern Physics: Conference Series, vol. 24, (2013).

[11]P Li, S B Wu, L N Wang,et al. Automatic recognition system of plate character based on machine vision, Metallurgical Industry Automation, vol. 37, no. 4, (2013), 12-15.

[12]V Novak, H Habiballa, et al. Recognition of damaged letters based on mathematical fuzzy logic analysis, Journal of Applied Logic, vol. 189, no. 2, (2014), 497-506. 